\title{
Effects of Body Weight Change on Development of Chronic Kidney Disease in Obese Metabolic Phenotypes
}

\author{
Akiko Toda Shigeko Hara Hiroshi Tsuji Yasuji Arase \\ Health Management Center, Toranomon Hospital, Minato-ku, Japan
}

\section{Keywords}

Chronic kidney disease · Obesity · Epidemiology · Obese metabolic phenotypes

\begin{abstract}
Introduction: Obesity is a risk factor for chronic kidney disease (CKD), but whether the reduction of body mass index (BMI) helps prevent CKD is controversial. Recently, obese metabolic phenotypes have raised considerable interest. We thus investigated the effect of BMI change on CKD development. Methods: We analyzed the data of 6,959 subjects who underwent annual health checkups in both 2013 and 2018. The subjects were categorized into five groups according to their $\mathrm{BMI}$ percentage change $(\triangle \mathrm{BMI})$ and classified into four obese metabolic phenotypes. By a multivariate logistic regression analysis, we investigated the association between $\mathrm{BMI}$ change and CKD development within the 5 years. $\boldsymbol{R e}$ sults: In total subjects, compared with the maintained BMI group ( $\triangle \mathrm{BMI} \geq 0 \%$ but $<2.5 \%$ ), the odd ratios (ORs) and $95 \%$ confidence intervals (Cls) of CKD development were 0.70 $(95 \% \mathrm{Cl} 0.54-0.91)$ for the severe $\mathrm{BMI}$ decrease group ( $\triangle \mathrm{BMI}$ $<-2.5 \%)$, and $1.40(95 \% \mathrm{Cl} 1.08-1.81)$ for the severe $\mathrm{BMI}$ increase group $(\triangle \mathrm{BMI} \geq 5 \%)$. In the metabolically healthy obese (MHO) phenotype, the risks of CKD development were significantly higher in the moderate $\mathrm{BMI}$ increase group ( $\triangle \mathrm{BMI}$ $\geq 2.5 \%$ but $<5 \%$ ) (OR $3.04,95 \% \mathrm{Cl} 1.19-7.78)$ and a severe BMI
\end{abstract}

increase group (OR 2.88, 95\% Cl 1.13-7.35). Regarding the metabolically unhealthy nonobese (MUNO) phenotype, the risks of CKD development were significantly lower in the severe $\mathrm{BMI}$ decrease group (OR $0.43,95 \% \mathrm{Cl} 0.25-0.74)$ and the moderate $\mathrm{BMI}$ decrease group ( $\triangle \mathrm{BMI} \geq-2.5 \%$ but $<0 \%$ ) (OR $0.58,95 \% \mathrm{Cl} 0.35-0.98)$. Conclusions: In the MHO phenotype, an increased BMI deteriorated CKD development, and a decreased BMI ameliorated CKD development in the MUNO phenotype.

(c) 2022 The Author(s).

Published by S. Karger AG, Basel

\section{Introduction}

Many studies have indicated that obesity is a risk factor for the development of chronic kidney disease (CKD) [1, 2]; however, the relationship between body mass index (BMI) change and CKD remains controversial [3]. Recently, in a subset of obese individuals, the obese metabolic phenotype including the metabolically healthy obese (MHO) was identified $[4,5]$. The MHO phenotype is characterized by the absence of metabolic abnormalities such as insulin resistance, dyslipidemia, and hypertension despite having excessive body fat. It remains controversial whether individuals with the MHO phenotype have a better prognosis and whether a weight change has some effects on their prognosis. Regarding CKD, MHO

Correspondence to:

Karger@karger.com www.karger.com/nef

Karger $\stackrel{\text { ' }}{5}$

GOPEN ACCESS
C 2022 The Author(s).

Published by S. Karger AG, Basel

This is an Open Access article licensed under the Creative Commons Attribution-NonCommercial-4.0 International License (CC BY-NC) (http://www.karger.com/Services/OpenAccessLicense), applicable to the online version of the article only. Usage and distribution for commercial purposes requires written permission. 
subjects have been reported to have a higher risk for CKD than metabolically healthy nonobesity (MHNO) subjects [6-9]. However, the association between CKD development and body weight (BW) change in the subject with MHO phenotype or other obese metabolic phenotypes has not been revealed. Herein, we investigated the association between CKD development and BMI change in the obese metabolic phenotype by using the data of annual health checkups.

\section{Materials and Methods}

\section{Study Population}

All of the subjects had undergone annual health checkups at the Health Management Center of Toranomon Hospital in Tokyo, Japan. The number of healthy subjects who had undergone annual health checkups in both 2013 and 2018 was 8,204. After excluding the data of 1,208 subjects who were diagnosed with CKD in 2013, 6 subjects who were pregnant, and 31 subjects whose data were defective, we performed a longitudinal study using the data of the remaining 6,959 subjects. The study was approved by the Ethics Committees of Toranomon Hospital. The requirement for informed consent was waived because we used deidentified retrospective data.

\section{Clinical and Laboratory Measurements}

Each subject's information was obtained through a self-administered questionnaire. The subject's weight and height were measured by our institution's staff, and the BMI was calculated using the following equation: BW $(\mathrm{kg}) /$ body height $(\mathrm{m})^{2}$. The subject's blood pressure was measured using a sphygmomanometer in a seated position. Blood samples were collected during hospital visits after an overnight fast. Serum high-density lipoprotein (HDL) cholesterol, serum low-density lipoprotein cholesterol, and serum creatinine were measured using the enzymatic method. Serum triglyceride was determined using a standardized enzymatic procedure for the glycerol phosphate oxidase assay. Fasting blood glucose was measured by the hexokinase-glucose-6-phosphate dehydrogenase method. Serum uric acid was measured by the uricase peroxidase method. The estimated glomerular filtration rate (eGFR) was calculated using a version of the Modification of Diet in Renal Disease equation modified for Japanese: eGFR (mL/ $\left.\mathrm{min} / 1.73 \mathrm{~m}^{2}\right)=194 \times$ age $^{-0.287} \times$ serum creatinine $(\mathrm{mg} / \mathrm{dL}$, the enzymatic method $)^{-1.094} \times(0.739$ if female $)$ [10]. BSA-unadjusted eGFR was calculated as follows: BSA-unadjusted eGFR $(\mathrm{mL} / \mathrm{min})$ $=$ BSA-adjusted eGFR $\left(\mathrm{mL} / \mathrm{min} / 1.73 \mathrm{~m}^{2}\right) \times$ BSA $\left(\mathrm{m}^{2}\right) / 1.73[11]$.

\section{Data Categorization}

The percentage change in the $\mathrm{BMI}(\triangle \mathrm{BMI})$ was calculated using the following equation: ([BMI in $2018-\mathrm{BMI}$ in 2013]/BMI in $2013) \times 100$. For our analysis, we classified the subjects into five groups based on the approximately quintile of their $\triangle \mathrm{BMI}$ value: (i) severe BMI decrease $(\triangle \mathrm{BMI}<-2.5 \%)(n=1,470)$; (ii) moderate BMI decrease $(\triangle \mathrm{BMI} \geq-2.5 \%$ but $<0 \%)(n=1,223)$; (iii) maintained BMI $(\triangle \mathrm{BMI} \geq 0 \%$ but $<2.5 \%)(n=1,748)$; (iv) moderate BMI increase $(\triangle \mathrm{BMI} \geq 2.5 \%$ but $<5 \%)(n=1,233)$; and $(\mathrm{v})$ severe BMI increase $(\Delta \mathrm{BMI} \geq 5 \%)(n=1,285)$.
Hypertension, dyslipidemia, hyperglycemia, hyperuricemia, and obesity were defined as follows. The subjects with systolic blood pressure $\geq 140 \mathrm{~mm} \mathrm{Hg}$, diastolic blood pressure $\geq 90 \mathrm{~mm} \mathrm{Hg}$, and/or being on medication for hypertension were categorized as having hypertension. Dyslipidemia was defined as a serum concentration of low-density lipoprotein cholesterol $\geq 140 \mathrm{mg} / \mathrm{dL}$, HDL cholesterol $<40 \mathrm{mg} / \mathrm{dL}$, triglyceride $\geq 150 \mathrm{mg} / \mathrm{dL}$, and/or being on medication for dyslipidemia. Hyperglycemia was defined as a serum concentration of fasting blood glucose $\geq 126 \mathrm{mg} / \mathrm{dL}$ and/ or being on medication for diabetes. Hyperuricemia was defined as a serum concentration of uric acid $>7.0 \mathrm{mg} / \mathrm{dL}$ and/or being on medication for hyperuricemia. A BMI value $\geq 25 \mathrm{~kg} / \mathrm{m}^{2}$ was classified as obesity following the criterion for the Asian population [12]. According to the K/DOQI-KDIGO guideline [13], an eGFR $<60 \mathrm{~mL} / \mathrm{min} / 1.73 \mathrm{~m}^{2}$ was defined as CKD.

Based on the BMI and metabolic factors, the obesity metabolic phenotypes were categorized $[14,15]$. Subjects who had less than two of the following four metabolic risk factors were considered metabolically healthy: (1) a systolic blood pressure $\geq 130 \mathrm{~mm} \mathrm{Hg}$ and/or a diastolic blood pressure $\geq 85 \mathrm{~mm} \mathrm{Hg}$ and/or being on medication for hypertension; (2) a serum concentration of triglyceride $\geq 150 \mathrm{mg} / \mathrm{dL}$, and/or being on medication for dyslipidemia; (3) a serum concentration of HDL cholesterol $<40 \mathrm{mg} / \mathrm{dL}$; and (4) a serum concentration of fasting blood glucose $\geq 100 \mathrm{mg} / \mathrm{dL}$ and/ or being on medication for diabetes. According to the criterion, the subjects were categorized into one of the four groups [5]: (I) MHNO: BMI $<25 \mathrm{~kg} / \mathrm{m}^{2}$ and $<2$ metabolic risk factors; (II) metabolically unhealthy nonobese (MUNO): BMI $<25 \mathrm{~kg} / \mathrm{m}^{2}$ and $\geq 2$ metabolic risk factors; (III) MHO: $\mathrm{BMI} \geq 25 \mathrm{~kg} / \mathrm{m}^{2}$ and $<2$ metabolic risk factors; and (IV) metabolically unhealthy obese (MUO): $\mathrm{BMI} \geq 25 \mathrm{~kg} / \mathrm{m}^{2}$ and $\geq 2$ metabolic risk factors.

\section{Statistical Analysis}

To compare the baseline characteristics among category groups, we performed one-way analyses of variance for values and $\chi^{2}$ tests for proportions. By $\chi^{2}$ tests for the trend, we compared the percentages of CKD development or the transition of metabolic status among category groups of BMI change. By multivariate logistic regression analyses, we calculated the odds ratios (ORs) and 95\% confidence intervals (CIs) for the development of CKD. In these analyses, age, sex, hypertension, dyslipidemia, hyperuricemia, and hyperglycemia were adjusted as CKD-related factors, and basal BMI and basal eGFR were adjusted for excluding the effects of the basal condition.

All statistical analyses were performed using SPSS ver. 25 software (SPSS IBM Inc., Chicago, IL, USA). A $p$ value $<0.05$ was considered statistically significant.

\section{Results}

\section{Study Population}

We analyzed the data of 6,959 subjects in a longitudinal study (Fig. 1). Almost all the subjects were Japanese. Their baseline characteristics are summarized in Table 1. We classified the subjects into four obese metabolic phenotypes (Table 2). It is logical that subjects with the MUO 


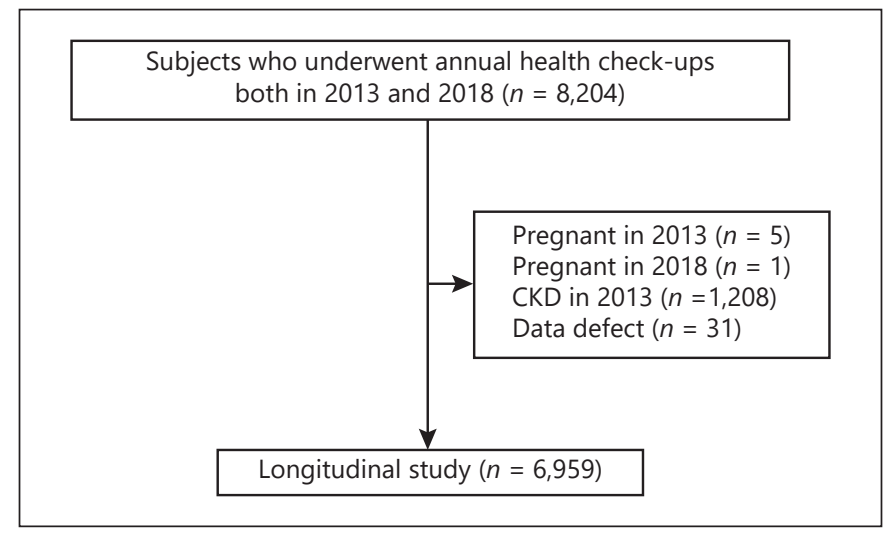

Fig. 1. Participants included and excluded from this study.

and the MHO phenotypes showed a higher average BMI value than those with the MUNO and MHNO phenotypes, and subjects with the MUO and MUNO phenotypes had worse metabolic conditions than those with the MHO and MHNO phenotypes. The subjects' age and eGFR, as well as the percentage of men, were significantly different among the four phenotypes $(p<0.001)$.

\section{Association of BMI Change with CKD Development}

The percentages of CKD development (number of patients with CKD development/total) in the total population and each obese metabolic phenotype of BMI change over 5 years are shown in Figure 2. In the total population, 759 subjects (10.9\%) developed CKD. The $p$ values by $\chi^{2}$ test for the trend were 0.006 in the total subjects, 0.23 in the MUO phenotype, $<0.001$ in the MUNO phenotype, 0.04 in the MHO phenotype, and 0.98 in the MHNO phenotype. In the total subjects, the subject with MUNO and subjects with $\mathrm{MHO}$ associations between BMI change and CKD development were significant.

To determine the precise association between BMI change and CKD development, we performed a multivariate logistic regression analysis in which the maintained BMI group was used as a reference (Table 3). As covariate factors, age, sex, hypertension, dyslipidemia, hyperuricemia, hyperglycemia, basal BMI, and basal eGFR were adjusted. In total subjects, the risk of CKD development in the severe BMI decrease group was significantly lower than that of maintained BMI group (OR $0.70,95 \%$ CI $0.54-0.91, p=0.008$ ). In the severe BMI increase group, the risk of CKD development was significantly higher (OR 1.40, 95\% CI 1.08-1.81, $p=0.01$ ). However, in both the moderate BMI decrease group and the moderate BMI increase group, there was no significant
Table 1. Characteristics of subjects

$\begin{array}{ll}\text { Subjects, } n & 6,959 \\ \text { Age, years } & 52.5 \pm 10.1 \\ \text { Men, \% } & 67.2 \\ \text { Obesity, \% } & 22.8 \\ \text { BMI, kg/m² } & 22.9 \pm 3.4 \\ \text { Systolic blood pressure, mm Hg } & 124.2 \pm 18.7 \\ \text { Diastolic blood pressure, mm Hg } & 78.2 \pm 11.4 \\ \text { Serum LDL cholesterol, } \mathrm{mg} / \mathrm{dL} & 119.4 \pm 29.3 \\ \text { Serum HDL cholesterol, } \mathrm{mg} / \mathrm{dL} & 61.8 \pm 15.7 \\ \text { Serum triglyceride, } \mathrm{mg} / \mathrm{dL} & 104.7 \pm 73.8 \\ \text { Serum uric acid, mg/dL } & 5.50 \pm 1.28 \\ \text { Serum fasting blood glucose, mg/dL } & 101.1 \pm 16.8 \\ \text { eGFR, mL/min/1.73 } \mathrm{m}^{2} & 76.2 \pm 11.2 \\ \text { Medication, \% } & \\ \quad \text { Hypertension } & 14.1 \\ \quad \text { Dyslipidemia } & 9.3 \\ \text { Hyperuricemia } & 4.5 \\ \text { Hyperglycemia } & 2.8\end{array}$

Continuous variables are expressed as mean \pm standard deviation. Categorical values are expressed as number or percentage. LDL, low-density lipoprotein.

association between the change of BMI and the development of CKD. By analysis using BSA-unadjusted eGFR, the result was similar (online suppl. Table 1; see www. karger.com/doi/10.1159/000522159 for all online suppl. material).

We also analyzed the relationship between BMI change and CKD development in subjects with each category of the obese metabolic phenotypes. In subjects with the MUNO phenotype, reduced CKD development was observed with a severe decrease and a moderate decrease in the BMI. The ORs were 0.43 (95\% CI 0.25-0.74, $p=0.002$ ) in the severe BMI decrease group and 0.58 (95\% CI $0.35-$ $0.98, p=0.04)$ in the moderate BMI decrease group. In subjects with $\mathrm{MHO}$ phenotype, showing a moderate or severe BMI increase after 5 years, CKD development was higher than that in subjects showing a maintained BMI. The ORs were 3.04 (95\% CI 1.19-7.78, $p=0.02$ ) in the moderate BMI increase group and 2.88 (95\% CI $1.13-$ $7.35 p=0.03)$ in the severe BMI increase group. In the subjects with the MUO or MHNO phenotype, no significant effects by BMI change on CKD development were observed.

\section{Transition of the Metabolic Condition}

Transitions of the metabolic condition after 5 years in MHO phenotype or MUNO phenotype were shown in Figure 3. In subjects with MHO phenotype, the percent- 
Table 2. Characteristic of subjects categorized into obese metabolic phenotypes

\begin{tabular}{llllll}
\hline & MUO & MUNO & MHO & MHNO & $p$ value \\
\hline Subjects, $n$ & 1,038 & 1,506 & 548 & 3,867 & \\
Age, years & $52.6 \pm 9.3$ & $57.2 \pm 10.1$ & $49.3 \pm 8.9$ & $51.1 \pm 9.9$ & $<0.001$ \\
Men, \% & 85.5 & 76.8 & 71.7 & 57.8 & $<0.001$ \\
BMI, kg/m ${ }^{\mathrm{a}}$ & $28.0 \pm 3.0$ & $22.4 \pm 1.8$ & $27.1 \pm 2.2$ & $21.2 \pm 2.1$ & $<0.001$ \\
Systolic blood pressure, mm Hg & $139.6 \pm 17.1$ & $135.4 \pm 17.1$ & $124.8 \pm 14.7$ & $115.7 \pm 14.8$ & $<0.001$ \\
Diastolic blood pressure, mm Hg & $87.4 \pm 10.4$ & $84.7 \pm 10.4$ & $78.4 \pm 9.1$ & $73.2 \pm 9.4$ & $<0.001$ \\
Serum LDL cholesterol, mg/dL & $123.8 \pm 29.6$ & $120.7 \pm 29.7$ & $125.1 \pm 30.5$ & $116.9 \pm 28.6$ & $<0.001$ \\
Serum HDL cholesterol, mg/dL & $50.7 \pm 12.7$ & $60.5 \pm 16.7$ & $56.8 \pm 12.4$ & $66.0 \pm 14.7$ & $<0.001$ \\
Serum triglyceride, $\mathrm{mg} / \mathrm{dL}$ & $166.6 \pm 119.7$ & $126.7 \pm 82.0$ & $100.1 \pm 41.4$ & $80.1 \pm 35.7$ & $<0.001$ \\
Serum uric acid, $\mathrm{mg} / \mathrm{dL}$ & $6.2 \pm 1.2$ & $5.7 \pm 1.2$ & $5.9 \pm 1.2$ & $5.2 \pm 1.2$ & $<0.001$ \\
Serum fasting blood glucose, mg/dL & $113.5 \pm 26.7$ & $108.7 \pm 16.1$ & $97.8 \pm 13.1$ & $95.2 \pm 9.7$ & $<0.001$ \\
eGFR, mL/min/1.73 m ${ }^{2}$ & $75.8 \pm 11.3$ & $74.8 \pm 10.4$ & $76.3 \pm 11.3$ & $76.9 \pm 11.4$ & $<0.001$ \\
Medication, \% & & & & & $<0.001$ \\
$\quad$ Hypertension & 33.7 & 28.7 & 7.5 & 4.1 & $<0.001$ \\
$\quad$ Dyslipidemia & 20.6 & 20.8 & 2.0 & 2.8 & $<0.001$ \\
$\quad$ Hyperglycemia & 7.5 & 5.1 & 1.6 & 0.7 & $<0.001$ \\
$\quad$ Hyperuricemia & 11.6 & 7.0 & 4.9 & 1.6 & \\
\hline
\end{tabular}

Continuous variables are expressed as mean \pm standard deviation. Categorical values are expressed as number or percentage. LDL, low-density lipoprotein. ${ }^{a}$ To compare among four categories, one-way analysis of variance for values and $x^{2}$ test for proportion were performed. $p$ value of less than 0.05 was considered to be statistically significant.

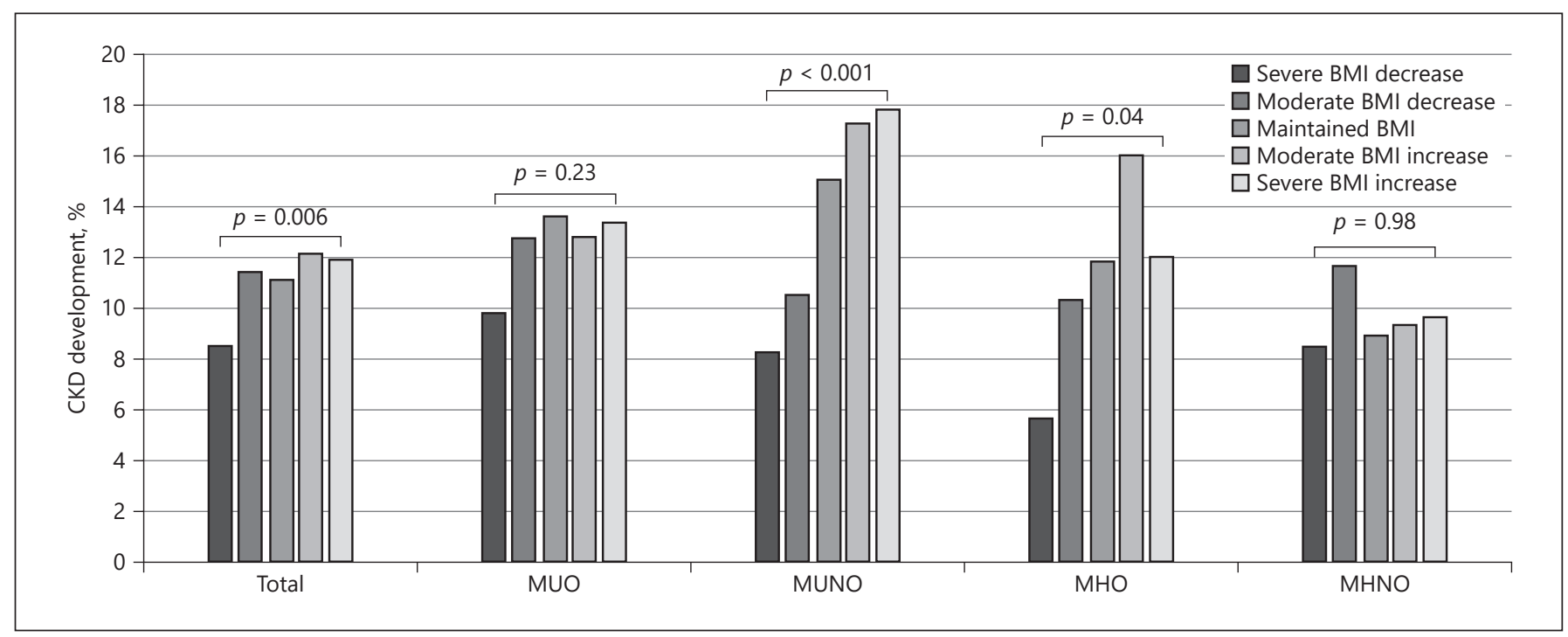

Fig. 2. CKD development after 5 years. The percentages of CKD development in total subjects or each group based on change in BMI were shown. The $p$ values were analyzed by the $\chi^{2}$ test for trend.

ages of a transition from metabolic healthy into metabolic unhealthy condition after 5 years were $23.0 \%$ in the severe BMI decrease group, $33.3 \%$ in the moderate BMI decrease group, $37.8 \%$ in the maintained BMI group,
$39.0 \%$ in the moderate BMI increase group, and $43.6 \%$ in the severe BMI increase group (Fig. 3a). The metabolic status showed a tendency to change from healthy into unhealthy condition with a deterioration of obesity. By a $\chi^{2}$ 
test for the trend, this tendency was significant $(p=0.012)$. In subjects with the MUNO phenotype, the percentages of a transition from a metabolic unhealthy into metabolic healthy condition after 5 years were $32.7 \%$ in the severe BMI decrease group, $29.6 \%$ in the moderate BMI decrease group, $22.9 \%$ in the maintained BMI group, $19.8 \%$ in the moderate BMI increase groups, and $15.2 \%$ in the severe BMI increase group (Fig. 3b). With a decrease in the BMI, the metabolic condition significantly shifted from unhealthy into healthy state $(p<0.001)$.

\section{Discussion}

The results of our longitudinal study in which the subjects were total population indicated that a severe BMI reduction had a significant effect on the prevention of CKD development, and a severe increase in the BMI was a risk factor for CKD development. Many studies have reported that obesity is associated with an increased risk of CKD development $[1,2,16]$. Interventions conducted to help individuals decrease their BMI values, which are thought to be beneficial for the prevention of the development of CKD. Several reports have described the beneficial effect of BMI decrease on renal function $[17,18]$, but other studies obtained opposite results $[19,20]$. One of the reasons for these inconsistent findings could be the diversity of individuals' condition. Recently, a unique phenotype of obese individuals displaying a favorable metabolic profile called $\mathrm{MHO}$ has been reported [21, 22]. Thus, we examined the association between BMI change and CKD development in each metabolic phenotype. Our results revealed that a deterioration of the obese state increased the risk of CKD development in subjects with $\mathrm{MHO}$, and a decrease in the BMI significantly reduced the risk of CKD development in the MUNO phenotype. In the subjects with MUO or MHNO, no significant effects by BMI change on CKD development were observed.

Although the MHO individuals could be expected to have a favorable clinical course, whether MHO individuals have a better prognosis remains debatable [23-25]. Concerning kidney function, several studies and metaanalyses reported that $\mathrm{MHO}$ subjects have a higher risk for incident CKD than MHNO subjects [6-9]. However, no reports have investigated the association between CKD development and BW change in MHO subjects.

The cause of the increased risk of CKD development by a deterioration of obese state may be that not a few MHO subjects transitioned to MUO over time. Several 


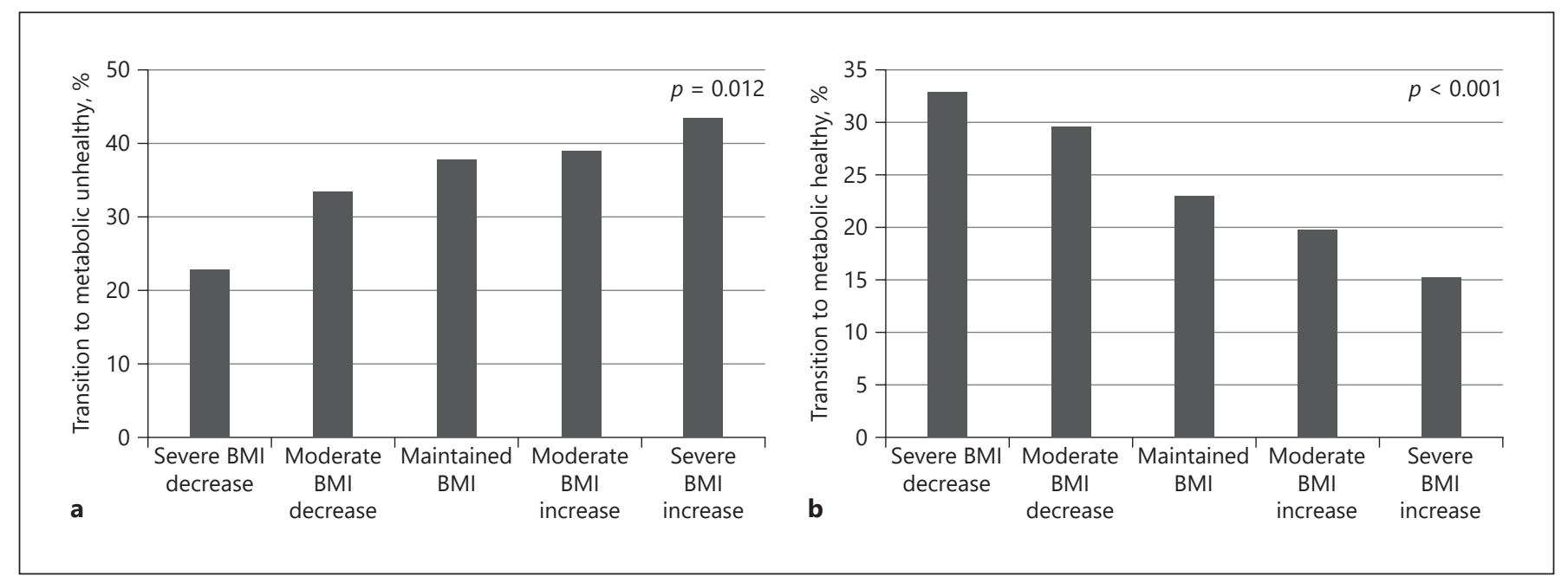

Fig. 3. The transition of metabolic condition. a In subjects with MHO in 2013, the percentages of the subjects with metabolic unhealthy in 2018 were shown in each group of BMI change. The $p$ values by the $\chi^{2}$ test for trend were 0.012. b In subjects with MUNO in 2013, the percentages of the subjects with metabolic healthy in 2018 were shown in each group of BMI change. The $p$ values by $\chi^{2}$ test for trend were $<0.001$.

reports presented that, in the baseline MHO group, subjects who converted to MHNO were not at an increased risk for CKD, whereas subjects who evolved to a metabolically unhealthy status had a high risk of CKD development $[8,26]$. In our subjects with MHO phenotype, a transition from a metabolic healthy to metabolic unhealthy condition was higher with an aggravation of obese. The deterioration of the metabolic condition caused by an increased BMI could be one of the reasons for the increased risk of CDK development. For clarifying the detailed mechanism, further investigation in a population with larger number of subjects is needed.

Few studies have reported a relationship between MUNO and CKD development. Our result presented that the risk of CKD development decreases with a reduction of BMI. One of the causes could also be a transition of the metabolic phenotype. In our subjects with the MUNO phenotype, a decrease of BMI tends to ameliorate the metabolic condition. The reduction in the risk of CKD development could be related to the improvement of the metabolic status. In subjects with the MUNO phenotype, despite their nonobese condition, a benefit of a BW reduction could be expected. However, because of a risk of healthy accident accompanied by BW loss, the losing BW should be performed under a doctor's supervision.

This study possesses several limitations. First, we did not have precise information about the reason for the change in BMI. Because the weights of bone and muscle generally remain constant in healthy adults, we speculate that the change in the BMI among our subjects might reflect a change in the percentage of fat. However, whether the BMI change is a result of a healthy status or an unhealthy status is essential for the prognosis. Thus, a prospective study in which BMI control is performed according to a program including intervention such as exercise and diet adjustment is necessary. Second, regarding the definition of CKD according to the K/DOQI guidelines [27], the continuation of an eGFR $<60 \mathrm{~mL} / \mathrm{min} / 1.73 \mathrm{~m}^{2}$ for 3 months is indispensable. However, we analyzed the data obtained from annual health checkups, and it was not possible to observe the subjects for several months. And we do not have the data about albuminuria at baseline. Thus, we used a definition of CKD based on a single measurement and only eGFR.

In conclusion, a deterioration of obesity increased the risk of CKD development in subjects with the MHO phenotype; a decreased BMI ameliorated the risk of CKD development in subjects with the MUNO phenotype. Our result supposed that the effect of BMI change on CKD development could be attributed to a change in metabolic condition. However, our analyses were based on the data of the general population and the appropriate BMI depends on patients. A control of BMI under a physician's supervision is indispensable. 


\section{Acknowledgments}

The authors are grateful to all of the participants, other physicians, medical staff, and other contributors to this study. This research did not receive any specific grant from funding agencies in the public, commercial, or not-for-profit sectors.

\section{Statement of Ethics}

This study was approved by the Ethics Committees at Toranomon Hospital (approval number 1521-K). The requirement for informed consent was waived because we used deidentified retrospective data.

\section{Conflict of Interest Statement}

The authors have no conflicts of interest to declare.

\section{Funding Sources}

No funding sources were used for the conduct of this study.

\section{Author Contributions}

Akiko Toda conceived and designed the work, analyzed the data, and wrote the manuscript. Shigeko Hara interpreted the result, contributed to the discussion, and reviewed the manuscript. Hiroshi Tsuji interpreted the result, contributed to the discussion, and reviewed the manuscript. Yasuji Arase interpreted the result, contributed to the discussion, and reviewed the manuscript. All authors gave important intellectual contributions, reviewed the manuscript, and provide final consent of the version to be published.

\section{Data Availability Statement}

The data that support the findings of this study are not publicly available due to their containing information that could compromise the privacy of research participants but are available from the corresponding author (A.T.) upon reasonable request.

\section{References}

1 Garofalo C, Borrelli S, Minutolo R, Chiodini P, De Nicola L, Conte G. A systematic review and meta-analysis suggests obesity predicts onset of chronic kidney disease in the general population. Kidney Int. 2017 May;91(5): 1224-35.

2 Lakkis JI, Weir MR. Obesity and kidney disease. Prog Cardiovasc Dis. 2018 Jul-Aug; 61(2):157-67.

3 Rhee CM, Ahmadi SF, Kalantar-Zadeh K. The dual roles of obesity in chronic kidney disease: a review of the current literature. Curr Opin Nephrol Hypertens. 2016 May;25(3):208-16.

4 Primeau V, Coderre L, Karelis AD, Brochu M, Lavoie ME, Messier V, et al. Characterizing the profile of obese patients who are metabolically healthy. Int J Obes. 2011 Jul;35(7):971-81.

5 Phillips CM. Metabolically healthy obesity: definitions, determinants and clinical implications. Rev Endocr Metab Disord. 2013 Sep; 14(3):219-27.

6 Jung CH, Lee MJ, Kang YM, Hwang JY, Kim EH, Park JY, et al. The risk of chronic kidney disease in a metabolically healthy obese population. Kidney Int. 2015 Oct;88(4):843-50.

7 Zhang J, Jiang H, Chen J. Combined effect of body mass index and metabolic status on the risk of prevalent and incident chronic kidney disease: a systematic review and meta-analysis. Oncotarget. 2017 May 30;8(22):35619-29.

8 Nam KH, Yun HR, Joo YS, Kim J, Lee S, Lee $\mathrm{C}$, et al. Changes in obese metabolic phenotypes over time and risk of incident chronic kidney disease. Diabetes Obes Metab. 2018 Dec;20(12):2778-91.
9 Alizadeh S, Esmaeili H, Alizadeh M, Daneshzad E, Sharifi L, Radfar H, et al. Metabolic phenotypes of obese, overweight, and normal weight individuals and risk of chronic kidney disease: a systematic review and meta-analysis. Arch Endocrinol Metab. 2019 Jul 29;63(4):427-37.

10 Imai E, Yasuda Y, Matsuo S. A decade after the KDOQI CKD guidelines: a perspective from Japan. Am J Kidney Dis. 2012 Nov; 60(5):729-30

11 Lopez-Martinez M, Luis-Lima S, Morales E, Navarro-Diaz M, Negrin-Mena N, Folgueras $\mathrm{T}$, et al. The estimation of GFR and the adjustment for BSA in overweight and obesity: a dreadful combination of two errors. Int J obes. 2020 May;44(5):1129-40.

12 Wen CP, David Cheng TY, Tsai SP, Chan HT, Hsu HL, Hsu CC, et al. Are Asians at greater mortality risks for being overweight than Caucasians? Redefining obesity for Asians. Public Health Nutr. 2009 Apr;12(4):497-506.

13 Levey AS, Eckardt KU, Tsukamoto Y, Levin A, Coresh J, Rossert J, et al. Definition and classification of chronic kidney disease: a position statement from Kidney Disease: Improving Global Outcomes (KDIGO). Kidney Int. 2005 Jun;67(6):2089-100.

14 Expert Panel on Detection, Evaluation, and Treatment of High Blood Cholesterol in Adults. Executive summary of the third report of the National Cholesterol Education Program (NCEP) Expert Panel on Detection, Evaluation, and Treatment of High Blood Cholesterol In Adults (Adult Treatment Panel III). JAMA. 2001 May 16;285(19):2486-97.
15 Yamagishi K, Iso $H$. The criteria for metabolic syndrome and the national health screening and education system in Japan. Epidemiol Health. 2017;39:e2017003.

16 Iseki K, Ikemiya Y, Kinjo K, Inoue T, Iseki C, Takishita S. Body mass index and the risk of development of end-stage renal disease in a screened cohort. Kidney Int. 2004 May;65(5): 1870-6.

17 Wakamatsu K, Seki Y, Kasama K, Uno K, Hashimoto K, Seto Y, et al. Prevalence of chronic kidney disease in morbidly obese Japanese and the impact of bariatric surgery on disease progression. Obes Surg. 2018 Feb; 28(2):489-96.

18 Lin YC, Lai YJ, Lin YC, Peng CC, Chen KC, Chuang MT, et al. Effect of weight loss on the estimated glomerular filtration rates of obese patients at risk of chronic kidney disease: the RIGOR-TMU study. J Cachexia Sarcopenia Muscle. 2019 Aug;10(4):756-66.

19 Ricardo AC, Anderson CA, Yang W, Zhang $\mathrm{X}$, Fischer MJ, Dember LM, et al. Healthy lifestyle and risk of kidney disease progression, atherosclerotic events, and death in CKD: findings from the Chronic Renal Insufficiency Cohort (CRIC) Study. Am J Kidney Dis. 2015 Mar;65(3):412-24.

$20 \mathrm{Ku} \mathrm{E}$, Kopple JD, Johansen KL, McCulloch CE, Go AS, Xie D, et al. Longitudinal weight change during $\mathrm{CKD}$ progression and its association with subsequent mortality. Am J Kidney Dis. 2018 May;71(5):657-65. 
21 Samocha-Bonet D, Dixit VD, Kahn CR, Leibel RL, Lin X, Nieuwdorp M, et al. Metabolically healthy and unhealthy obese: the 2013 Stock Conference report. Obes Rev. 2014 Sep; 15(9):697-708.

22 Beh S. Is metabolically healthy obesity a useful concept? Diabet Med. 2019 May;36(5):53945.

23 Jung $\mathrm{CH}$, Lee WJ, Song KH. Metabolically healthy obesity: a friend or foe? Korean J Intern Med. 2017 Jul;32(4):611-21.
24 Phillips CM. Metabolically healthy obesity across the life course: epidemiology, determinants, and implications. Ann N Y Acad Sci. 2017 Mar; 1391(1):85-100.

25 Smith GI, Mittendorfer B, Klein S. Metabolically healthy obesity: facts and fantasies. J Clin Invest. 2019 Oct 1;129(10):3978-89.
26 Cho YK, Lee J, Kim HS, Park JY, Lee WJ, Kim YJ, et al. Impact of transition in metabolic health and obesity on the incident chronic kidney disease: a nationwide cohort study. J Clin Endocrinol Metab. 2020 Mar 1;105(3): dgaa033.

27 Inker LA, Astor BC, Fox CH, Isakova T, Lash JP, Peralta CA, et al. KDOQI US commentary on the 2012 KDIGO clinical practice guideline for the evaluation and management of CKD. Am J Kidney Dis. 2014 May;63(5):71335. 